\title{
Impact of mindfulness on the neural responses to emotional pictures in experienced and beginner meditators
}

\author{
Véronique A. Taylor ${ }^{a}$, Joshua Grant ${ }^{\mathrm{a}, \mathrm{b}, \mathrm{e}}$, Véronique Daneault ${ }^{\mathrm{a}}$, Geneviève Scavone ${ }^{\mathrm{a}}$, Estelle Breton ${ }^{\mathrm{a}}$, \\ Sébastien Roffe-Vidal a , Jérôme Courtemanche a , Anaïs S. Lavarenne ${ }^{\text {a }}$, Mario Beauregard a,b,c,d,* \\ a Centre de Recherche en Neuropsychologie et Cognition (CERNEC), Département de Psychologie, Université de Montréal, Montréal, Québec, Canada \\ b Centre de Recherche en Sciences Neurologiques, Département de Physiologie, Université de Montréal, Montréal, Québec, Canada \\ c Département de Radiologie, Université de Montréal, Montréal, Québec, Canada \\ ' Centre de recherche du Centre hospitalier de l'Université de Montréal (CRCHUM), Montréal, Québec, Canada \\ e Max Planck Institute for Human Cognitive and Brain Sciences, Department of Social Neuroscience, Leipzig, Germany
}

\section{A R T I C L E I N F O}

\section{Article history:}

Received 30 November 2010

Revised 26 May 2011

Accepted 1 June 2011

Available online 12 June 2011

\begin{abstract}
A B S T R A C T
There is mounting evidence that mindfulness meditation is beneficial for the treatment of mood and anxiety disorders, yet little is known regarding the neural mechanisms through which mindfulness modulates emotional responses. Thus, a central objective of this functional magnetic resonance imaging study was to investigate the effects of mindfulness on the neural responses to emotionally laden stimuli. Another major goal of this study was to examine the impact of the extent of mindfulness training on the brain mechanisms supporting the processing of emotional stimuli. Twelve experienced (with over $1000 \mathrm{~h}$ of practice) and 10 beginner meditators were scanned as they viewed negative, positive, and neutral pictures in a mindful state and a non-mindful state of awareness. Results indicated that the Mindful condition attenuated emotional intensity perceived from pictures, while brain imaging data suggested that this effect was achieved through distinct neural mechanisms for each group of participants. For experienced meditators compared with beginners, mindfulness induced a deactivation of default mode network areas (medial prefrontal and posterior cingulate cortices) across all valence categories and did not influence responses in brain regions involved in emotional reactivity during emotional processing. On the other hand, for beginners relative to experienced meditators, mindfulness induced a down-regulation of the left amygdala during emotional processing. These findings suggest that the long-term practice of mindfulness leads to emotional stability by promoting acceptance of emotional states and enhanced present-moment awareness, rather than by eliciting control over low-level affective cerebral systems from higher-order cortical brain regions. These results have implications for affect-related psychological disorders.
\end{abstract}

(c) 2011 Elsevier Inc. All rights reserved.

\section{Introduction}

Originating from ancient Eastern traditions (Nataraja, 2008), meditation is an increasingly prominent object of study within the fields of clinical psychology and behavioral neuroscience. Mindfulness is a form of meditation which has been operationally defined as "a kind of nonelaborative, nonjudgmental, present-centered awareness in which each thought, feeling, or sensation that arises in the attentional field is acknowledged and accepted as it is" (Bishop, 2004). The detached observation state which is adopted during mindfulness is thought to promote an objective and adaptive manner of responding to emotional triggers in contrast to a habitual pattern of

\footnotetext{
* Corresponding author at: Mind/Brain Research Lab (MBRL), Centre de Recherche en Neuropsychologie et Cognition (CERNEC), Département de Psychologie, Université de Montréal, C.P. 6128, succursale Centre-Ville, Montréal, Québec, Canada H3C 3J7. Fax: + 1 5143435787.

E-mail address: mario.beauregard@umontreal.ca (M. Beauregard).
}

emotional reactivation typically driven by past experiences, fears and preconceptions (Bishop, 2004). Consistent with this view, it has been demonstrated that mindfulness-based clinical interventions have beneficial outcomes on the treatment of affect-related psychopathology, including major depression (Bondolfi et al., 2010; Teasdale et al., 2000) and anxiety disorders (Goldin and Gross, 2010; Kabat-Zinn et al., 1992; Kim et al., 2009). However, there still remains much to uncover on the specific mechanisms as well as neural substrates through which this state of awareness influences emotional processes.

To date, a few studies have examined the effects of meditation on the processing of emotional stimuli. Focused-breathing typically practiced during mindfulness has been shown to promote emotional self-regulation by reducing reactivity to emotionally laden pictures (positive and negative) (Arch and Craske, 2006). Mindfulness meditation practiced following a sad mood induction was also shown to decrease dysphoric mood states (Broderick, 2005). Additionally, Brefczynski-Lewis et al. (2007) found a negative correlation between the number of hours of meditation training and 
right amygdala activation during concentration meditation in a group of experienced meditators while processing negative emotional sounds. In another study (Farb et al., 2010), the MBSR program led to reduced activation in regions associated with autobiographical memory and increased activity in the right insula (Farb et al., 2010). Finally, Lutz et al. (2008) investigated the effects of compassion meditation on the processing of emotional auditory stimuli. They found increased right insular activation for experienced meditators, relative to beginners, during a state of compassion meditation while listening to negative emotional sounds (compared to emotionally positive sounds). This brain region plays an important role in awareness of interoceptive states (Craig, 2004; Critchley et al., 2004). Compassion meditation also induced increased amygdalar activation while listening to emotional and neutral sounds for experienced meditators relative to beginners. It is noteworthy that since compassion meditation involves maintaining a non-referential state of compassion, different brain processes may be involved in mindfulness meditation during emotional processing as opposed to compassion meditation.

An increasing number of neuroimaging studies have investigated the neural substrates underlying emotional self-regulation strategies, such as cognitive distancing and reappraisal (Beauregard et al., 2001; Levesque et al., 2003; Ochsner et al., 2002, 2004). These strategies have commonly been associated with the recruitment of prefrontal cortical areas critically involved in executive control functions (e.g., lateral prefrontal cortex [LPFC], medial prefrontal cortex [MPFC], and anterior cingulate cortex $[\mathrm{ACC}])$, and decreased activation in subcortical structures implicated in emotional processing (e.g., amygdala) (Beauregard et al., 2001; Levesque et al., 2003; Ochsner et al., 2002, 2004).

Convergent with neuroimaging studies of emotional self-regulation, there are some reports that meditative states are associated with increased activation in various prefrontal cortical areas. Such heightened prefrontal activation has been interpreted as reflecting increased recruitment of attentional resources during meditation (Newberg et al., 2001). Interestingly, mindfulness was shown to be associated with increased MPFC/LPFC activation and reduced amygdalar activation during affect labeling, which is commonly employed during meditation in order to identify emotional states (Creswell et al., 2007). In this study, Creswell et al. (2007) found that participants high in trait mindfulness also had negative correlations between areas of the PFC (MPFC and LPFC) and the right amygdala, but not those low in trait mindfulness. These findings are consistent with evidence that the MPFC, through its inhibitory downstream connections to the amygdala, is implicated in the extinction of conditioned fear as well as the dampening of negative affect (Amaral et al., 1992; Davidson, 1998). Similar results were also found in clinical populations. For example, Goldin and Gross (2010) reported that patients with social anxiety disorder (SAD) exhibited decreased right amygdalar activation after viewing phrases of negative self-beliefs, and increased activation in regions relevant to the control of attention (including regions of the parietal cortex) after having completed an 8-week mindfulness-based stress reduction program (MBSR). Goldin and Gross (2010) interpreted these results as indicating that mindfulness meditation helps to reduce avoidance-related behavior to threatening stimuli in SAD patients by enhancing the recruitment of brain regions involved in attentional control.

However, from a phenomenological perspective, it should be noted that controlled modulation and reappraisal differ to a large extent from mindfulness. Indeed, most emotional self-regulation strategies aim at altering emotional states, whereas mindfulness is based on accepting emotional states as they are (Kabat-Zinn, 1994). Consistent with this view, some neuroimaging studies suggest that long-term mindfulness meditation training can lead to decreased activation in prefrontal cortical areas, particularly the MPFC (Farb et al., 2007; Grant et al., 2011). In one of these studies, Farb et al. (2007) found that an experiential focus condition (monitoring the self in terms of present-moment circumstances) was associated with deactivations of the ventral and dorsal MPFC compared with a narrative self-focus task (monitoring the self in terms of self-descriptive traits). This effect was more pronounced and widespread in individuals having completed an 8-week MSBR program compared to a control group. A similar pattern of results has recently been reported in Zen meditators experiencing emotionally salient painful stimuli (Grant et al., 2011). In this study, when normally attending to painful stimulations, experienced meditators exhibited decreased activation in several brain regions involved in executive control and emotional appraisal, including the MPFC, LPFC, and amygdala compared to nonmeditators. These decreased activations found in executive control areas have been proposed to reflect that meditation training is associated with a reduction in cognitive elaboration of aversive stimuli (Grant et al., 2011).

Clearly, however, methodological discrepancies in meditative and control tasks, as well as the different strategies or cognitive processes employed and the level or type of expertise in meditators may account for the inconsistencies in the literature. Nevertheless, the premise that meditation stabilizes emotion by not evaluating or appraising salient stimuli is in line with the prefrontal deactivations reported during mindfulness (Farb et al., 2007; Grant et al., 2011). Yet to date, little is known regarding the brain mechanisms through which mindfulness meditation modulates the processing of emotional stimuli.

In this context, a central goal of the present functional magnetic resonance imaging (fMRI) study was to investigate the neural mechanisms mediating the effect of this form of meditation on the processing of emotionally laden stimuli. Another major goal of this study was to examine the impact of the extent of mindfulness meditation experience on the brain mechanisms supporting the processing of emotional stimuli. Thus, a group of experienced meditators and a group of beginner meditators were scanned while they viewed negative, positive, or neutral pictures in a mindful state and a regular (non-mindful) state of awareness. They also rated stimuli on the emotional intensity they experienced when viewing the pictures. We hypothesized that the emotional stimuli viewed during mindfulness would be perceived as less emotionally intense than those viewed in a non-mindful state of awareness. Moreover, we hypothesized that mindfulness would lead to decreased activation in cerebral structures involved in emotional processing, such as the amygdala. Based on previous research (Brefczynski-Lewis et al., 2007), this was predicted to occur to a greater extent in experienced meditators relative to beginners. Finally, due to the lack of evaluative processes maintained during mindfulness meditation, we hypothesized that mindfulness would lead to reduced activation in prefrontal cortical areas, and that this would occur to a greater extent for the group of experienced meditators.

\section{Methods}

\section{Participants}

Before being selected for the study, potential participants underwent preliminary telephone screening and were not included if they had any current or previous psychiatric or neurological disorders, consumed any psychotropic drugs, or had any severe medical conditions. The group of experienced meditators consisted of 12 individuals with more than $1000 \mathrm{~h}$ of experience in Zen meditation ( 7 females, 5 males; 11 right-handed, 1 left-handed; 25-60 years of age, $M=46, S D=11$ ), and were recruited from meditation centers located in Montreal (Quebec, Canada). One participant from this group had 45 $000 \mathrm{~h}$ of meditation practice experience, and deviated in the number of hours of experience from the rest of the group (which ranged from 1000-3000 h, $M=1709, S D=694)$. Therefore, the analyses reported 
below were also conducted without the inclusion of this participant, and the results remained essentially unchanged. Given this, to avoid losing statistical power, this participant was kept in the statistical analyses.

The group of beginner meditators consisted of 10 individuals (4 females, 6 males; 10 right-handed, 1 ambidextrous, 1 left-handed; $22-54$ years of age, $M=34, S D=12$ ) with no prior experience in meditation or similar practices such as yoga. There were no significant between-group differences with respect to age $(p>0.05)$ or to the ratio of male/female participants $(p>0.05)$. The beginner meditators were recruited from Université de Montréal and the Centre de Recherche de l'Institut Universitaire de Gériatrie de Montréal (CRIUGM) through advertisement posters. They were given detailed instructions on how to practice mindfulness meditation based on various sources (Ricard, 2008; Kabat-Zinn, 1994; Thich Nhat Hanh, 1994). They were also given a written record of these instructions as well as a compact disc on which a guided mindfulness meditation session was recorded by the experimenters. Based on this guided session, beginners were instructed to practice mindfulness meditation 20 min per day, for 7 days before the fMRI experiment. During this week of training, the experimenters followed up with participants to ensure that they understood how to practice meditation and to verify that they had been completing their practice daily. Compliance was followed up upon by a telephone interview, once in the middle of the week of training, during which participants were asked if they had questions. Before beginning their training period, participants were given the experimenters' contact information and were welcomed to ask any questions. Only one individual had two days of meditation practice prior to testing (due to scheduling restraints), but reported compensating accordingly by practicing additional time each day. In all other participants, $100 \%$ compliance was observed. All participants reported having successfully understood the mindfulness exercises. Participants were remunerated $\$ 50$ for their participation in this study. They all gave written informed consent and the study was approved by the ethics research committee of the CRIUGM.

\section{Stimuli and experimental procedure}

Blood oxygen level dependent (BOLD) signal changes were measured while participants viewed pictures in a 'normal' state (i.e., without attempting to modulate attention; this condition is referred to as the 'Baseline' condition) and in a mindful state (during which they were instructed to mindfully attend to the stimuli; this condition is referred to as the 'Mindful' condition). Two runs were acquired per condition. To prepare themselves to view the pictures in a mindful state of awareness (Mindful condition), participants were instructed to relax and focus on their breath (for approximately 1-2 min) until they felt they had attained a state of mindfulness. Before the start of the experiment, in order for participants to be comfortable to practice mindfulness within a scanner environment, participants were given some practice trials in a mock scanner, which simulated the physical environment. Participants were given approximately 20 practice trials in the Baseline condition, and 20 practice trials in the Mindful condition. Before the start of the runs in the Baseline condition, participants were told that they were not required to meditate during the upcoming session. The Baseline and Mindful conditions alternated between runs, but the order in which the conditions were completed was counterbalanced across participants (Mindful-Baseline-Mindful-Baseline and Baseline-Mindful-Baseline-Mindful). The stimuli consisted of a total of 216 pictures selected from the International Affective Picture System ([IAPS] Lang et al., 2005). An event-related design was implemented. Each run consisted of 54 trials with 18 trials for each emotional category. Within each run and for each participant, the pictures were presented in a randomized manner. Valence and arousal ratings published by Lang et al. (2005) for positive, negative, and neutral pictures were equated across conditions (negative pictures: va- lence $=3.00 \pm 0.76$, arousal $=5.78 \pm 0.86$ for the Baseline condition, valence $=3.00 \pm 0.79$, arousal $=5.79 \pm 0.78$ for the Mindful condition; neutral pictures: valence $=4.94 \pm 0.32$, arousal $=2.90 \pm 0.45$ for the Baseline condition, valence $=4.89 \pm 0.26$, arousal $=2.86 \pm 0.48$ for the Mindful condition; positive pictures: valence $=7.20 \pm 0.45$, arous$\mathrm{al}=4.96 \pm 1.11$ for the Baseline condition, valence $=7.21 \pm 0.52$, arousal $=5.06 \pm 0.98$ for the Mindful condition). The task design was based on a previous neuroimaging study of emotional self-regulation (Ochsner and Gross, 2005). Each trial consisted of the following events: a cue was first presented for $2 \mathrm{~s}$ in the centre of a screen ('Baseline' or 'Mindful'), indicating the state of awareness in which to attend to the upcoming picture. Next, a positive, negative or neutral picture was presented for $6 \mathrm{~s}$, after which a rating scale appeared for participants to rate the strength of their emotional state induced by the stimuli $(0=$ no emotion was felt, 1 = weak emotion, $2=$ moderate emotion, $3=$ strong emotion, $4=$ very strong emotion) using a five-button response box. Finally, there was a brief rest period until the beginning of the next trial during which the word 'Relax' appeared for a variable time (1-3 s) in the center of the screen. The duration of the rest period between stimuli was randomized throughout each run, in order to prevent participants from anticipating the onset of subsequent stimuli. Each run lasted approximately $10 \mathrm{~min}$. Participants were explicitly instructed to watch the screen at all times. Stimulus presentation and response selection were controlled by the program E-Prime (version 1.0, Psychology Software Tools, Inc.) running on a separate portable computer. Stimuli were projected via a projector, through a lense onto a rear-projection screen that was attached in the magnet bore at the level of the neck. Participants viewed the stimuli on a slanted mirror placed inside the head coil.

To examine the effects of mindfulness on the magnitude of the subjective emotional responses experienced while viewing the stimuli, a Condition $\times$ Valence $\times$ Group mixed measures ANOVA was performed. The responses from two beginner participants were excluded, one because responses had been rated according to valence, and the other due to response box recording failure during scanning. Thus, a total $n=20$ (12 experienced, 8 beginners) cases were included in these analyses. For each condition, the mean response for pictures in each emotional category was computed. The assumptions for conducting a mixed-measure ANOVA, such as homogeneity of variance between groups and linearity, were met. All variables were normally distributed, with skewness and kurtosis values ranging from -1.5 to 1.5 . No univariate or multivariate outliers were detected. Due to response box failure on certain trials, some stimuli were missing emotional intensity ratings (with no more than $25 \%$ of participant ratings missing for any given stimulus), which were distributed at random across groups. These missing values were replaced with the group's corresponding mean value to avoid losing statistical power. The Greenhouse-Geisser correction was used to correct for sphericity of repeated measures. Statistical significance was set at an alpha level of .05. Self-report data were analyzed using the SPSS package (Version 17.0).

\section{fMRI data acquisition and analysis}

MRI was performed using a whole-body 3.0 Tesla MRI system (Magnetom Trio, Siemens Electric, Erlangen, Germany) located at the Unité de Neuroimagerie Fonctionnelle (UNF) of the CRIUGM. Thirty-five contiguous slices (voxel size $=3 \mathrm{~mm} \times 3 \mathrm{~mm} \times 3.5 \mathrm{~mm}$ ) were acquired in an inclined axial plane. These $\mathrm{T}^{*}$ weighted functional pictures were acquired using a two-dimensional echo-planar-imaging pulse sequence (repetition time $[\mathrm{TR}]=2500 \mathrm{~ms}$, echo time $[\mathrm{TE}]=40 \mathrm{~ms}$, flip angle $=90^{\circ}$, matrix size $=64 \times 64$ voxels). For each participant, a high resolution anatomical scan was also performed (three-dimensional, spoiled gradient echo sequence; 176 slices, slice thickness $=1 \mathrm{~mm}$, $\mathrm{TR}=19 \mathrm{~ms}, \mathrm{TE}=4.92 \mathrm{~ms}$, flip angle $=25^{\circ}$; matrix size $=256 \times 256$ voxels). The anatomical scan was acquired after the first two functional scans were completed. 
fMRI data were analyzed using Statistical Parametric Mapping software (SPM8, Wellcome Department of Cognitive Neurology, London, UK). Two participants in each group only had data for two runs (one from each condition) due to testing constraints or excessive movement; however, the number of degrees of freedom was adjusted accordingly in the group analyses. The first three volumes of each run were excluded from the analyses to eliminate any $\mathrm{T} 2 *$-equilibrium effects. For each participant, images were motion corrected and spatially normalized into an MRI stereotactic space (Talairach and Tournoux, 1988). Volumes deviating in translation by more than $3 \mathrm{~mm}$, which only occurred at the very beginning or very end of a given session, were considered outliers and were excluded from the analyses. Images were spatially smoothed with a Gaussian kernel of $8 \mathrm{~mm}$ at fullwidth half maximum to improve the signal-to-noise ratio, and to accommodate for residual variations in functional neuroanatomy that usually persist between participants after spatial normalization. For the statistical analyses, the time series of the images were convolved with a canonical hemodynamic response function, and effects at each voxel were estimated using the general linear model. For each run, the onsets for the three categories of pictures (negative, neutral, and positive) were included into the model. In addition, the six motion parameters $(x$, $y, z$, pitch, roll, and yaw) were entered as regressors of no interest in order to remove unspecific residual activation patterns related to movement (Friston et al., 1996). For each participant, a fixed-effect model was used to compute specific contrast pictures. At the grouplevel, contrast images for each condition and emotional category were entered into a full factorial Condition $\times$ Valence $\times$ Group ANOVA randomeffect model. Global normalization was carried out by including the global value (i.e. the average BOLD signal intensity at all within-brain voxels across time) from each participant as a nuisance variable. Unless otherwise specified, statistical parametric maps were first thresholded at $p<.001$ uncorrected for multiple comparisons. Then, an a priori search strategy was conducted to detect activation loci by performing small volume corrections in the regions of interest (ROIs). The ROIs encompassed the amygdala, insula (Brodmann area [BA] 13 and 14), putamen, caudate nucleus, hippocampus, dorsal (BA 24 and 32) and rostro-ventral (BA 24 and 25) ACC, MPFC (BA 9 and 10), LPFC (BA 9 and 10 ), and OFC (BA 11 and 47). These brain regions have been found to be activated on a more or less consistent basis in previous functional neuroimaging studies of emotional processing and self-regulation (Phan et al., 2002). For this a priori search, a cluster-level threshold of $p<0.05$ corrected for multiple comparisons at the family-wise error rate (FWE) was used. Clusters exceeding a threshold of $p<0.05$ FWE corrected at the whole-brain level are also reported. The predefined ROIs were located using masks created with the program MARINA (@ Bertram Walter, 2002, Bender Institute of Neuroimaging, University of Glessen, Germany). A separate mask was created for each ROI, and small volume searches were performed for each region separately, within their own mask. Only clusters showing a spatial extent of at least 5 contiguous voxels were kept for image analysis. To aid in the clarity of the presentation of the results, "Mindfulness-induced activations" refer to increased activity observed in the Mindful relative to Baseline condition, and "Mindfulness-induced deactivations" refer to decreased activity observed in the Mindful compared to the Baseline condition. Cohen's $D(d)$ effect sizes were also examined for all significant loci of activation reported (by transforming $t$-maps into effect size maps using the Volumes toolbox implemented in SPM8).

\section{Results}

\section{Self-report data}

The ANOVA revealed a significant main effect of Valence $(F(2,18)=$ $\left.122.39, p<.001, \eta^{2}=.87\right)$. Negative pictures $(p<.0001, M=2.08$; $S D=0.65)$ and positive pictures $(p<.001, M=1.64 ; S D=0.67)$ were rated as more emotionally intense than neutral pictures $(M=0.52$;
$S D=0.41)$. A significant main effect of Condition $(F(1,18)=7.23$, $p<.05, \eta^{2}=.29$ ) was also found. This effect was due to the fact that pictures viewed in the Mindful condition $(M=1.29 ; S D=0.57)$ were rated as less emotionally intense than those viewed at Baseline $(M=1.53 ; S D=0.59)$.

No significant effect of Group $(F(1,18)=0.63, p=.438)$ was found. In addition, the Group $\times$ Condition $(F(1,18)=0.72, p=.382)$, Group $\times$ Valence $\times$ Condition $(F(1,18)=0.45, p=.747)$, or Valence $\times$ Condition $\left(F(1,18)=1.94, p=.17, \eta^{2}=.170\right)$ interactions were not significant. Nonetheless, to examine whether mindfulness had the same impact within each emotional category, simple effects of Condition (one-way repeated measures ANOVAs) were computed within each emotional picture category (collapsing across groups since no main effect or interactions with this factor were found). These analyses revealed a significant main effect of mindfulness on the processing of negative pictures $(F(1,18)=6.23, p=.022$, $\left.\eta^{2}=.25\right)$, such that negative pictures viewed in a state of mindfulness were rated as less intense $(M=1.93, S D=0.65)$ than negative pictures viewed in a normal state $(M=2.16, S D=0.71)$. A similar effect was found for positive pictures $(F(1,18)=6.23, p=.015$, $\eta^{2}=.25 ; M=2.79, S D=0.64$ for the Baseline condition; $M=2.43$, $S D=0.71$, for the Mindful condition). For neutral pictures, a significant effect of mindfulness was also found $(F(1,18)=5.40$, $p=.031, \eta^{2}=.22 ; M=0.62, S D=0.49$ for the Baseline condition; $M=0.44, S D=0.37$, for the Mindful condition), but this result may have been due to the little variability observed for neutral pictures, and thus, very small mean differences between conditions may have been found to be significant. These results are shown in Fig. 1.

\section{fMRI data}

\section{Emotional processing at Baseline}

When subtracting neutral from negative pictures in the Baseline condition, experienced meditators exhibited BOLD signal increases (two-sample $t$-test) in the LPFC (BA 9), MPFC (BA 9), OFC (BA 11/47), rostro-dorsal ACC (BA 32/24), anterior insula (BA 14), hippocampus, caudate nucleus, putamen, left amygdala and, though at a more liberal threshold ( $p<.005$ uncorrected), right amygdala. Beginner meditators showed BOLD signal increases in the MPFC (BA 9), LPFC (BA 9), left rostro-dorsal ACC (BA 32), hippocampus, OFC (BA 47), anterior insula (BA 14), and, though at a more liberal threshold $(p<.007)$, the amygdala. No between-group differences were observed for this contrast.

Additionally, when subtracting neutral from positive pictures in the Baseline condition, experienced meditators had BOLD signal increases (two-sample $t$-test) in the left LPFC (BA 9), MPFC (BA 9), left OFC (BA 47), hippocampus, left putamen, and left amygdala. Other loci of activation were found in the right rostro-dorsal ACC (BA 32), right caudate nucleus, and left anterior and posterior insula (BA 13/14). As for the beginner meditators, BOLD signal increases were noted in the rostro-dorsal ACC (BA 24/32), LPFC (BA 9), MPFC (BA 9), right anterior insula (BA 14), left anterior and posterior insula (BA 13/14), OFC (BA 47), PCC (BA 31), caudate nucleus, putamen, hippocampus, and amygdala. The only between-group difference for this contrast was a greater activation in the rostro-ventral ACC (BA 25 ) for beginner relative to experienced meditators.

\section{Brain activity related to Mindfulness and Group}

\section{Condition $\times$ Valence $\times$ Group interactions}

Specific contrasts were computed with regard to the interactions for which we had a priori hypotheses, namely contrasts comparing between-group differences in Mindfulness vs. Baseline conditions. Thus, two separate models were created: one for negative emotional processing by subtracting negative from neutral pictures and examining differences between conditions as well as groups (negative 

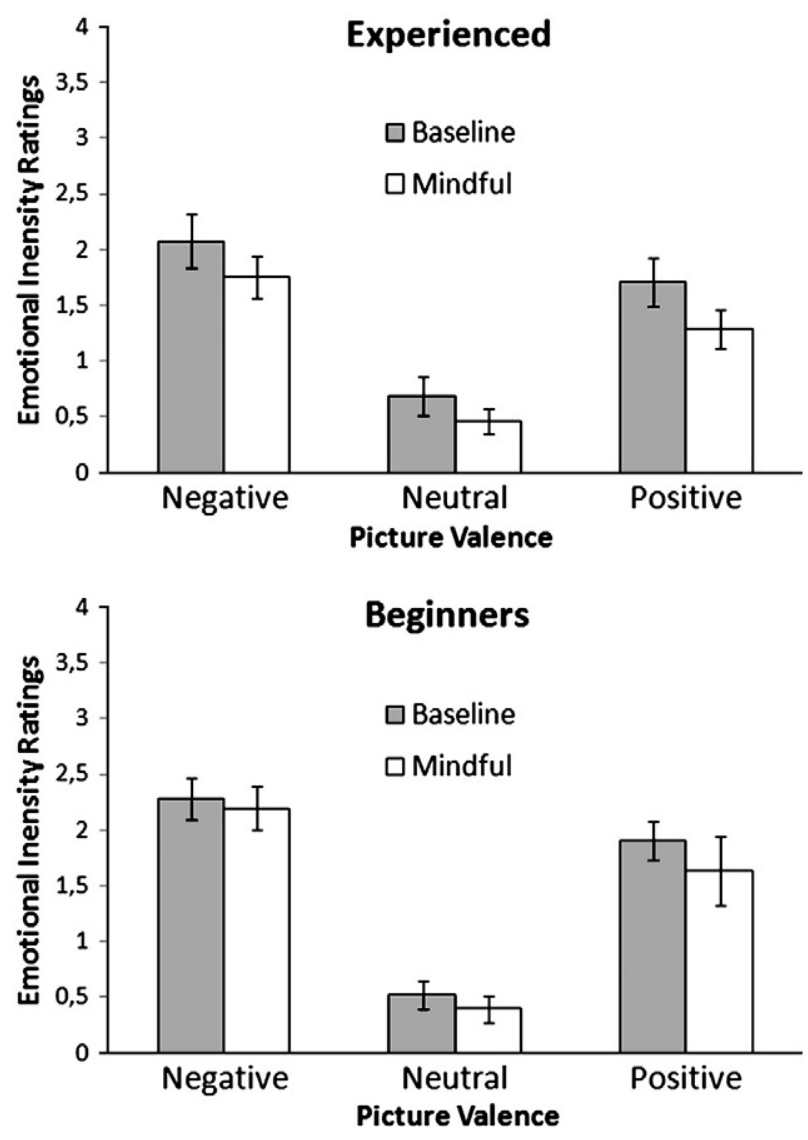

Fig. 1. Emotional intensity ratings (shown on the y-axis) by Group and Condition for each Valence category of pictures (x-axis). Emotional intensity elicited from viewing the pictures was rated on a scale of 0 to $4(0=$ no emotion, $1=$ weak emotion, $2=$ moderate emotion, $3=$ strong emotion, $4=$ very strong emotion). Mindfulness significantly reduced emotional intensity scores across Groups and Valence categories. No main effect of Group nor interactions were found. Means are shown with standard error bars.

minus neutral pictures, Mindful vs. Baseline, experienced vs. beginners), and the other for positive emotional processing by subtracting positive from neutral pictures and examining differences between conditions as well as groups (positive minus neutral pictures, Mindful vs. Baseline, experienced vs. beginners). These results are illustrated in Fig. 2.

Negative emotional processing. During negative emotional processing, Mindfulness induced deactivations - for beginners relative to experienced meditators - in the left amygdala $(k=16, t=2.96$, $Z=2.90, x=-27, y=-1, z=-18, P(\mathrm{FWE})=.055, d=0.54)$. No Mindfulness-induced deactivations during negative emotional processing were observed when contrasting experienced vs. beginner meditators.

Mindfulness-induced activations and deactivations were also explored within each group, revealing mindfulness-induced deactivations (at a more liberal threshold of $p<0.005$ uncorrected) in the left amygdala $(k=22, t=3.82, Z=3.70, x=-27, y=-1, z=-18$, $P(\mathrm{FWE})=.010, d=0.70)$ and right amygdala $(k=13, t=3.53$, $Z=3.43, x=27, y=-1, z=-16, P(\mathrm{FWE})=.012, d=0.65)$ for beginners, but not for experienced meditators. For experienced meditators, mindfulness-induced deactivations during negative emotional processing were observed (at a more liberal threshold of $p<0.005)$ in the left LPFC (BA 9/46, $k=33, t=3.33, Z=3.25, x=-36$, $y=30, z=15, P$ (uncorr.) $=.001, d=0.61$ ), though this was a sub- threshold trend. With respect to mindfulness-induced activations, no significant loci were observed within each group and betweengroups.

Positive emotional processing. During positive emotional processing, mindfulness induced deactivations (at a more liberal threshold of $p<0.005$ uncorrected) in the left amygdala for beginners vs. experienced meditators (Table 1 ). No mindfulness-induced deactivations during positive emotional processing were noted when contrasting experienced vs. beginner meditators.

Within-group analyses revealed that for beginners, but not for experienced meditators, mindfulness induced deactivations in the amygdala, right posterior insula (BA 13), putamen, left anterior insula (BA 13), caudate nucleus, left hippocampus, and right ACC (BA 24). No mindfulness-induced deactivations were observed for experienced meditators. During positive emotional processing, no mindfulnessinduced activations between or within groups were noted.

\section{Condition $\times$ Group interaction}

To examine more general effects induced by mindfulness, i.e. across Valence categories, between-group differences in the Condition $\times$ Group interaction were explored. Thus, contrasts comparing between-group activations for the Mindful vs. Baseline conditions across all Valence categories were computed. Mindfulness-induced deactivations were found in the right MPFC (BA 10, $k=51, t=3.75$, $Z=3.63, x=11, y=53, z=11, P(\mathrm{FWE})=.040, d=0.69)$ and right PCC (BA 29, $k=10, t=3.34, Z=3.26, x=10, y=-48, z=17, P$ $(\mathrm{FWE})=.023, d=0.61)$ for experienced relative to beginners.

Mindfulness-induced activations and deactivations across Valence categories were also examined within each group separately. For experienced meditators, mindfulness induced a deactivation of the right MPFC (though this was a sub-threshold trend at a more liberal threshold of $p<.005$ ) (BA 10, $k=36, t=3.14, Z=3.07, x=13, y=49$, $z=9, P$ (uncorr.) $=.001, d=0.58$ ) in experienced, but not in beginner meditators. No mindfulness-induced activations were found for experienced meditators.

For beginners, but not experienced meditators, mindfulness induced increased activity in the right medial frontal gyrus (BA 8, $k=486$, $t=4.86, Z=4.63, x=29, y=20, z=40, P(\mathrm{FWE})=.011, d=0.89)$, the left medial frontal gyrus (BA $8, k=218, t=3.98, Z=3.85, x=-26$, $y=22, z=43, P(\mathrm{FWE})=.007, d=0.85)$, the right PCC (BA 31, $k=84$, $t=4.34, Z=4.17, x=11, y=-50, z=22, P(\mathrm{FWE})=.006, d=0.80)$, the right inferior parietal lobule (IPL; BA 39, $k=460, t=4.57, Z=4.37$, $x=38, y=-64, z=25, P$ (FWE corrected at whole-brain level $)=.011$, $d=0.84$ ), and the left superior occipital gyrus/IPL (BA 19/39, $k=532$, $t=4.27, Z=4.11, x=-29, y=-69, z=26, P$ (FWE corrected at whole-brain level) $=.006, d=0.78$ ). Mindfulness did not induce any deactivations in beginners across Valence categories (Fig. 3).

\section{Main effects}

The main effects of interest and of relevance to this study, namely the main effect of Condition across Groups and Valence categories, as well as the main effect of Group across conditions and emotional categories, were also examined.

Condition. When collapsing across Groups and Valence categories, no mindfulness-induced deactivations were observed. However, mindfulness induced activations were observed, at a threshold of $p<.005$ uncorrected, in the right MPFC/LPFC (BA 8, $k=258, t=3.83, z=3.71$, $x=31, y=20, \mathrm{z}=38, P(\mathrm{FWE})=.055, d=0.67)$ and left MPFC/LPFC (BA 8, $k=231, t=3.63, z=3.53, x=-26, y=22, z=43$, P (FWE) $=$ $.070, d=0.70)$. These activations seemed to have been driven by the group of beginners, as they were situated in the same loci as those found within the group of beginners alone, and were not present in the group of experienced meditators alone. 

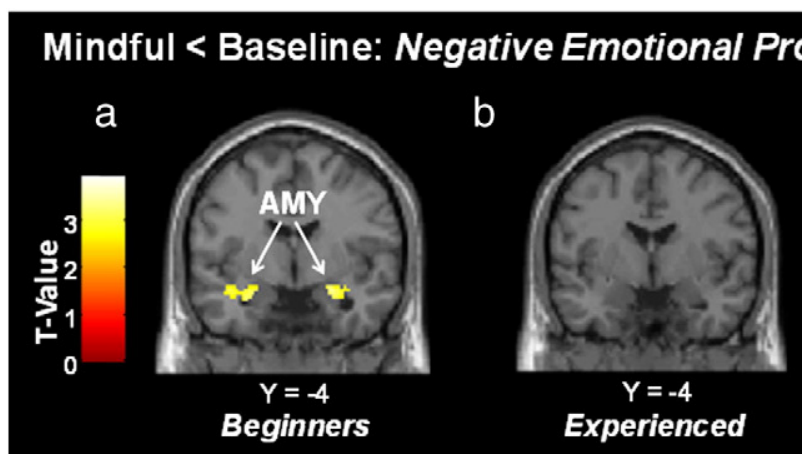

\section{essing}

$\mathrm{C}$

c

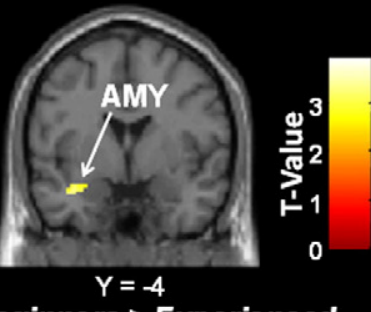

Beginners > Experienced
LAMY

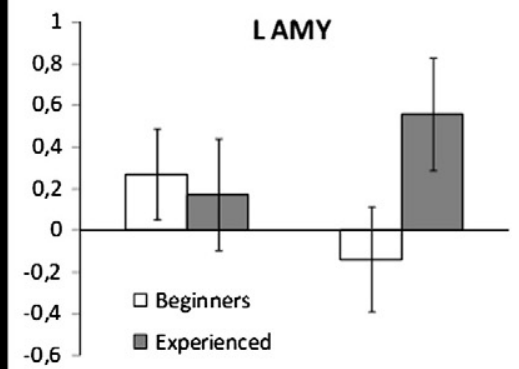

Baseline: Pos $>$ Neu Mindful: Pos $>$ Neu

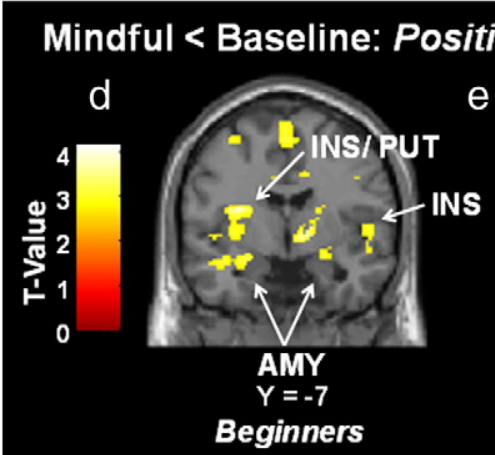

e NS
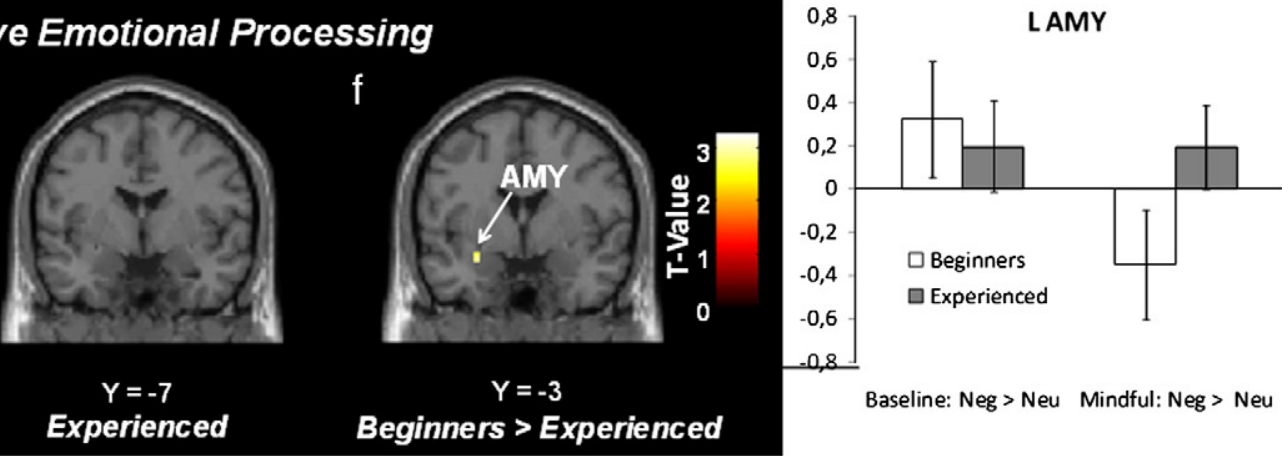

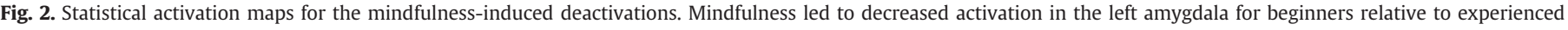

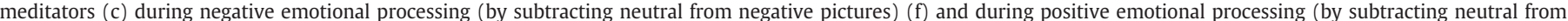

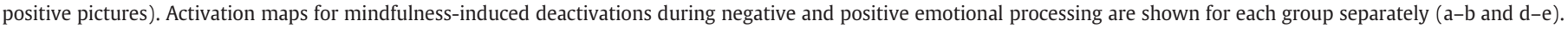

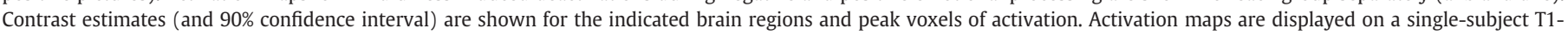
weighted template image included in the SPM8 software package. AMY, amygdala; PUT, putamen; INS, insula.

\section{Group}

When examining group differences by collapsing across Conditions and Valence categories, beginners exhibited increased activity in the left lingual gyrus (BA 18/17), left fusiform gyrus (BA 37), left OFC (BA 47), right hippocampus, PCC (BA 29/31), and thalami (Table 2).
Experienced meditators did not exhibit any increased activations relative to beginners for this contrast.

In sum, mindfulness induced a deactivation of the amygdala during the processing of negative and positive emotional stimuli for beginners relative to experienced meditators. Additionally,

Table 1

Mindfulness-induced deactivations: positive vs. neutral pictures.

\begin{tabular}{|c|c|c|c|c|c|c|c|c|c|}
\hline \multirow[t]{2}{*}{ Brain regions } & \multirow[t]{2}{*}{ BA } & \multirow[t]{2}{*}{ \# voxels } & \multirow[t]{2}{*}{$t$-value } & \multirow[t]{2}{*}{$Z$-value } & \multicolumn{3}{|c|}{ Coordinates } & \multirow[t]{2}{*}{$p$} & \multirow[t]{2}{*}{$d^{\mathrm{a}}$} \\
\hline & & & & & $\mathrm{x}$ & $\mathrm{y}$ & z & & \\
\hline \multicolumn{10}{|l|}{ Beginners } \\
\hline $\mathrm{R}$ posterior INS & 13 & 579 & 4.26 & 4.02 & 29 & -27 & 21 & $.004^{\mathrm{b}}$ & 0.76 \\
\hline L putamen & & 335 & 4.15 & 4.00 & -20 & 3 & -11 & $.035^{\mathrm{b}}$ & 0.61 \\
\hline R putamen/AMY/thalamus & & 395 & 4.08 & 3.94 & 18 & 6 & -4 & $.020^{\mathrm{b}}$ & 0.75 \\
\hline L amygdala & & 31 & 4.07 & 3.93 & -22 & -1 & -14 & $.009^{\mathrm{c}}$ & 0.75 \\
\hline $\mathrm{R}$ amygdala & & 5 & 3.43 & 3.34 & 24 & -5 & -11 & $.015^{\mathrm{c}}$ & 0.63 \\
\hline L putamen/anterior INS & 13 & 66 & 3.84 & 3.72 & -29 & -9 & 12 & $.023^{\mathrm{c}}$ & 0.71 \\
\hline L putamen/caudate nucleus & & 28 & 3.71 & 3.60 & -13 & 6 & 5 & $.027^{\mathrm{c}}$ & 0.70 \\
\hline R Caudate Nucleus & & 53 & 3.83 & 3.71 & 8 & 3 & 10 & $.017^{\mathrm{c}}$ & 0.68 \\
\hline L hippocampus & & 10 & 3.60 & 3.50 & -33 & -13 & -15 & $.045^{\mathrm{c}}$ & 0.66 \\
\hline R ACC & 24 & 20 & 3.27 & 3.20 & 8 & 12 & 27 & $.046^{\mathrm{c}}$ & 0.60 \\
\hline
\end{tabular}

Experienced

No mindfulness-induced deactivations were found for Experienced meditators.

Beginners $>$ Experienced

L AMY

3.03

2.97

$-24$

$-14$

$.057^{\mathrm{C}}$

0.56

Experienced $>$ Beginners

No mindfulness-induced deactivations were observed for Experienced vs. Beginner meditators.

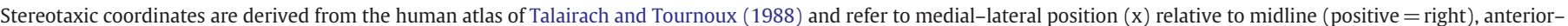

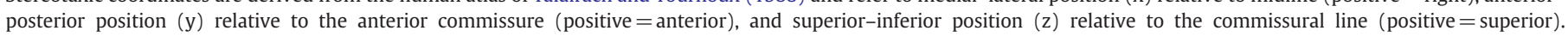
Designations of Brodmann areas for cortical areas are also based on this atlas. BA, Brodmann area, AMY, amygdala; INS, insula; ACC, anterior cingulate cortex; L, left; R, right.

a Cohen's $D$ measure of effect size.

b Corrected cluster-wise $p$ value (FWE) at whole-brain level.

c Corrected cluster-wise $p$ value (FWE) within small volume correction. 


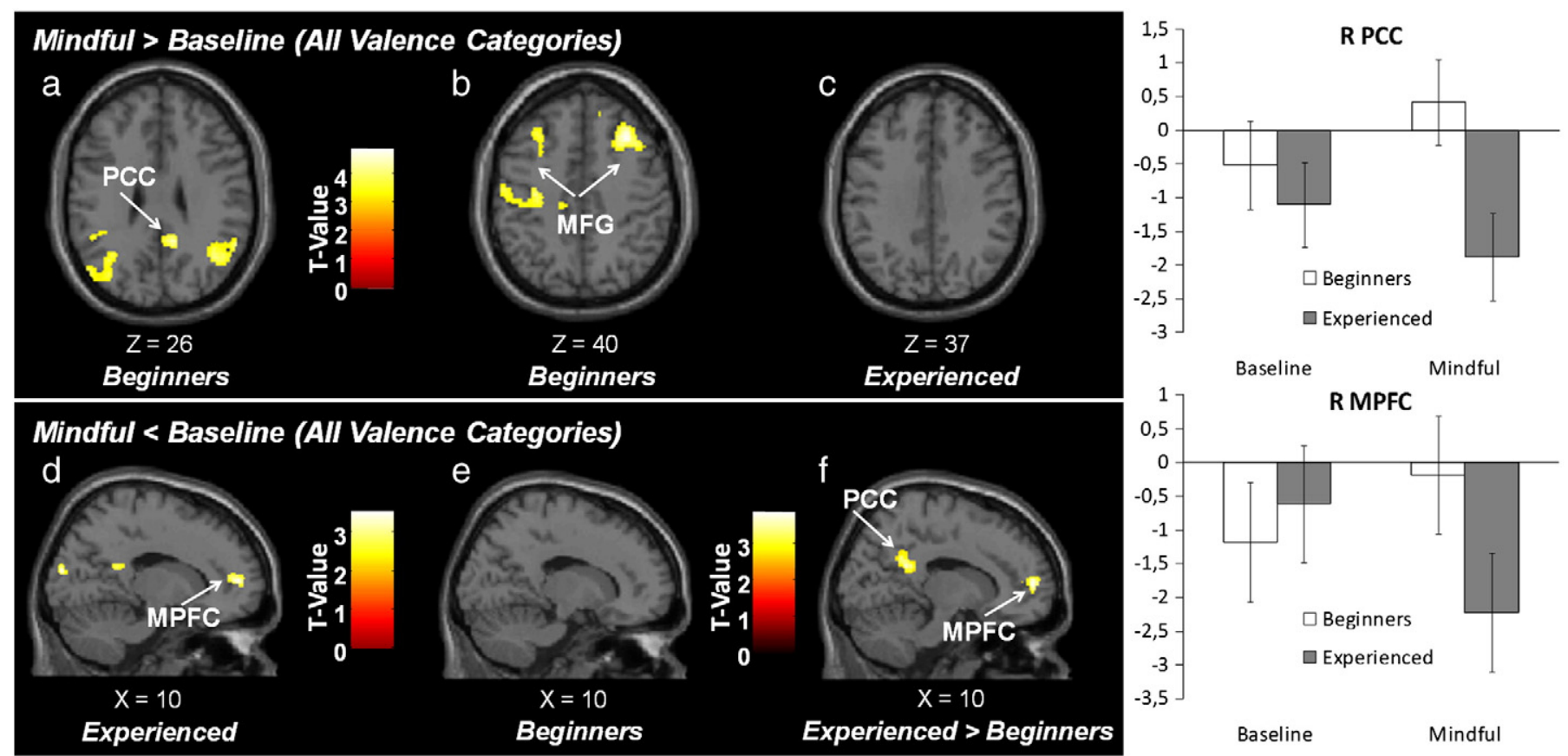

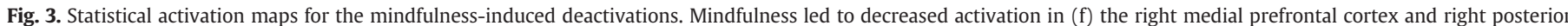

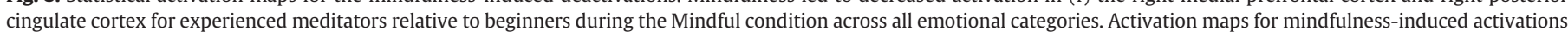

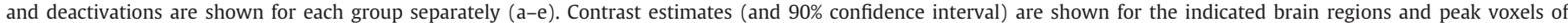
activation. MPFC, medial prefrontal cortex; PCC, posterior cingulate cortex; MFG, medial frontal gyrus.

mindfulness induced decreased activity in the MPFC (BA 10) and PCC (BA 29) activity across all Valence categories for experienced meditators relative to beginners.

Finally, for all activation loci reported, Cohen's $D$ effect sizes $(d)$ ranged from 0.54 to 1.24 , which constitute medium to large effects according to established benchmarks (small: 0.20, medium: 0.50, large: 0.80; Cohen, 1992). Regression analyses with the number of hours of meditation practice on the contrasts reported above were also computed, but no significant activations for either group, nor between groups were found, perhaps due to the relatively small sample size and the heterogeneous range of meditation experience within the group of experienced meditators.

\section{Discussion}

The results of the present study can be summarized as follows Behavioral response data revealed that mindfulness attenuated emotional intensity perceived from all valence categories of pictures across the entire sample of participants, whereas functional brain imaging data indicated that this attenuation was achieved via distinct neural mechanisms for each group. For experienced meditators relative to beginners, mindfulness induced deactivations of regions involved in the default mode network (right MPFC and right PCC) across valence categories of pictures, but did not influence brain activity in emotional reactivity brain areas during emotional

Table 2

Brain activations related to the main effect of group.

\begin{tabular}{|c|c|c|c|c|c|c|c|c|c|}
\hline \multirow[t]{2}{*}{ Brain regions } & \multirow[t]{2}{*}{ BA } & \multirow[t]{2}{*}{ \# voxels } & \multirow[t]{2}{*}{$t$-value } & \multirow[t]{2}{*}{ Z-value } & \multicolumn{3}{|c|}{ Coordinates } & \multirow[t]{2}{*}{$p$} & \multirow[t]{2}{*}{$d^{\mathrm{a}}$} \\
\hline & & & & & $\mathrm{x}$ & $\mathrm{y}$ & $\mathrm{z}$ & & \\
\hline \multicolumn{10}{|c|}{ Beginners $>$ Experienced (across all Conditions and Valence categories) } \\
\hline L lingual gyrus & $18 / 17$ & 1172 & 6.75 & 6.20 & -22 & -79 & -6 & $<.001^{\mathrm{b}}$ & 1.24 \\
\hline L fusiform gyrus & 37 & 322 & 5.47 & 5.16 & -20 & -40 & -20 & $.040^{\mathrm{b}}$ & 1.13 \\
\hline L OFC & 47 & 13 & 3.97 & 3.84 & -27 & 26 & -8 & $.056^{\mathrm{c}}$ & 1.01 \\
\hline L PCC & 29 & 18 & 3.77 & 3.65 & -12 & -48 & 11 & $.016^{\mathrm{c}}$ & 1.00 \\
\hline R PCC & 31 & 6 & 3.51 & 3.41 & 12 & -54 & 24 & $.017^{\mathrm{c}}$ & 0.81 \\
\hline R hippocampus & & 59 & 4.92 & 4.68 & 24 & -31 & -7 & $.016^{\mathrm{c}}$ & 0.90 \\
\hline L thalamus & & 59 & 3.50 & 3.41 & -6 & -23 & 4 & $.013^{\mathrm{c}}$ & 0.64 \\
\hline $\mathrm{R}$ thalamus & & 23 & 4.20 & 4.05 & 17 & -30 & 4 & $.026^{\mathrm{c}}$ & 0.77 \\
\hline $\mathrm{R}$ thalamus & & 56 & 3.51 & 3.42 & 17 & -23 & 11 & $.014^{\mathrm{C}}$ & 0.62 \\
\hline
\end{tabular}

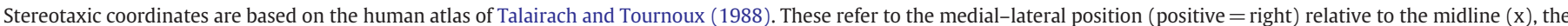

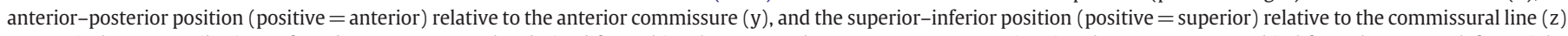

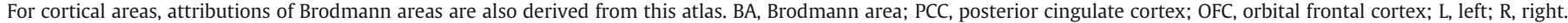

a Cohen's $D$ measure of effect size.

b Corrected cluster-wise $p$ value (FWE) within small volume corrections.

c Corrected cluster-wise $p$ value (FWE) at whole-brain level. 
processing. On the other hand, for beginners relative to experienced meditators, mindfulness induced a down-regulation of the left amygdala, a key emotional processing cerebral structure, during negative and positive emotional processing. Essentially, these findings indicate that mindfulness constitutes an efficient strategy to promote emotional stability. In addition, the results of this study suggest that with extensive meditation training, the mindfulness-induced emotional intensity attenuations may be achieved by accepting and fully experiencing emotional responses in the present moment without interference from internal thought and self-referent processes, as opposed to directly controlling low-order affective brain systems from higher-order cortical regions.

\section{Mindfulness and subjective emotional intensity elicited by the stimuli}

The attenuating effects of mindfulness on subjective emotional intensity observed in the present study are consistent with the view that mindfulness-based strategies promote emotional stability and constitute an efficient means of emotional self-regulation (Arch and Craske, 2006; Broderick, 2005). Indeed, the present findings suggest that mindful awareness fosters a more adaptive, relaxed, and objective manner of responding to emotional situations as opposed to habitual, automatic, and conditioned reaction patterns. This would be consistent with evidence that the practice of mindfulness is associated with increased well-being (Wallace and Shapiro, 2006), and that emotional stability is a significant predictor of happiness (Hills and Argyle, 2001).

Furthermore, our results are in line with evidence that mindfulness is beneficial for individuals with anxiety-related clinical disorders and traits (Baer, 2003). As such, individuals with generalized anxiety disorder tend to respond to negative mood challenges with greater emotional intensity relative to their control counterparts; they also tend to become overwhelmed by their emotions and understand them poorly (Mennin et al., 2005). Similarly, individuals high in neuroticism are more reactive (physiologically and subjectively) to negative and positive emotional stimuli than individuals who are emotionally stable (Norris et al., 2007). It has been suggested that anxious people engage in maladaptive responses to negative emotional challenges, such as avoidance, because the emotions they experience are too intense (Mennin et al., 2005). Thus, mindfulness may decrease the propensity to avoid processing emotions by attenuating the emotional intensity perceived from given events, and constitute an adaptive alternative coping mechanism for populations with anxiety-related traits or psychopathology.

Surprisingly, the effect of mindfulness did not apply specifically to any valence category or to either group (i.e., no significant interactions were found between condition, valence, or group). First, the fact that a mindful state of awareness attenuated emotional intensity perceived from positive pictures and did not specifically apply to negative pictures may reflect the fact that mindfulness endogenously enhances mood states (Zeidan et al., 2010; Wallace and Shapiro, 2006). As a result, mindfulness may diminish the need to seek out satisfaction from external positive stimuli, thereby attenuating the emotional intensity perceived from this category of pictures. Second, the fact that the attenuating effect of mindfulness also applied to neutral pictures may have been due to the little variability observed for this category of pictures, and thus, very small mean differences may have attained significance.

Finally, the fact that group differences in emotional intensity ratings failed to reach statistical significance may imply that mindfulness constitutes an efficient emotional self-regulation strategy for both highly experienced meditators and individuals having practiced mindfulness meditation for a short period of time. Nevertheless, the ease at which the state of mindfulness is achieved, as well as the frequency at which it is called upon, is likely quite different for experienced meditators as opposed to beginners. Since the ratings from two participants in the beginners' group were lost due to technical issues, an interaction between Group and Condition may have failed to attain statistical significance due to reduced power. Consequently, these results should be interpreted with caution until being replicated in larger participant samples. However, although both experienced and beginner meditators showed effects of mindfulness on subjective emotional intensity, differences in brain response patterns suggest that the two groups were in fact processing the stimuli in distinct ways during the Mindful condition.

Brain patterns related to mindfulness in experienced meditators and beginners

In keeping with one of our hypotheses and previous research (Farb et al., 2007), mindfulness was associated with decreased activation in the right MPFC (BA 10) in experienced meditators relative to beginners. This reduced activation in the right MPFC is consistent with findings from Bærentsen et al. (2010), who reported deactivation of this cortical region during sustained meditation. Additionally, our findings are in line with a study conducted by Kross and colleagues (Kross et al., 2009) investigating the relationship between brain function and the acceptance of emotional states. When participants were asked to recall a negative autobiographical experience and accept their emotional states (i.e., recognizing that their emotional responses were temporary passing states, which were distant from them and did not control them), decreased MPFC (BA 10) and subgenual ACC (BA 25) activations were observed. In contrast, when subjects were asked to 'ruminate' about their emotional states (i.e., to explicitly sustain focus on their emotional responses), they exhibited increased activity in these regions. Finally, when they were instructed to analyze their emotional responses (by analyzing the reasons underlying their affective responses in an objective manner), they did not exhibit any activity pattern changes in these regions.

Thus, the greater MPFC (BA 10) deactivation observed here in experienced relative to beginner meditators during the Mindful condition may reflect greater acceptance of affective states in response to the presented stimuli, as well as disengagement of appraisal and thought-related processes for individuals with extensive meditation experience. Such a process would be consistent with descriptions of mindfulness meditation as involving acceptance of sensations, emotions, and thoughts rather than attempting to control or change them (Bishop, 2004). The same interpretation, that is, reduced elaboration and appraisal, was recently put forth by Grant et al. (2011), who found a similar pattern of MPFC deactivation for meditators during pain perception, an inherently emotional experience. Future studies specifically examining the cognitive processes underlying prefrontal deactivations during meditation are needed to corroborate these hypotheses.

Furthermore, the mindful condition led to the deactivation of another brain area, the PCC (BA 29), for experienced relative to beginner meditators. This is consistent with the fact that this brain region, together with the MPFC, is a core structure of the so-called default mode network (DMN) (Buckner et al., 2008). The DMN consists of a set of spatially remote regions with coherent temporal dynamics, and is thought to reflect self-referential thought processes and daydreaming in the absence of explicit goal-directed tasks (Buckner et al., 2008). Thus, the greater deactivation observed in these two key DMN regions in experienced meditators (relative to beginners) is consistent with the findings from a previous study (Brefczynski-Lewis et al., 2007). In long-term practitioners of meditation, Brefczynski-Lewis et al. (2007) found that during a state of concentration meditation (compared to a baseline state of rest), there was a negative correlation between the number of hours of meditation experience and activation in DMN regions (MPFC and $\mathrm{PCC}$ ) in response to emotional and non-emotional sounds. This 
finding was reflected as greater distractibility elicited by the auditory stimuli in more inexperienced meditators.

Additionally, a recent brain imaging study found that depressed individuals failed to deactivate several DMN structures during the reappraisal and passive viewing of negative emotional pictures (Sheline et al., 2009). This finding was interpreted as reflecting increased interference from internally generated thoughts and rumination processes. In line with this, mindfulness meditation has been shown to reduce the occurrence of ruminative thoughts in remitted depressed patients (Ramel et al., 2004). Thus, the greater mindfulness-induced deactivation of the MPFC and PCC measured here - in experienced meditators relative to beginners - may reflect an adaptive process through which present-moment awareness is enhanced in individuals with long-term meditation experience, and information in the environment is processed with reduced distractibility and interference from self-referent thought or ruminative processes.

In contrast with what we found in experienced meditators during the Mindful condition across Valence categories, mindfulness induced increased activity in a superior portion of the medial frontal gyrus (BA 8 ) in beginners. This finding was reflected in the main effect of condition collapsed across groups. This result is compatible with the greater recruitment of a similar prefrontal cortical region previously reported in unexperienced meditators (compared with long-term practitioners of meditation) during a state of concentration meditation while listening to emotional and non-emotional sounds (BrefczynskiLewis et al., 2007). This increased prefrontal cortical activation detected for beginner meditators, relative to experienced meditators, resembles to a notable extent brain activation patterns found during voluntary regulation of emotion (Beauregard et al., 2001; Levesque et al., 2003; Ochsner et al., 2002; Ochsner and Gross, 2005). For instance, down-regulation of negative IAPS pictures through reappraisal has been reported to be associated with increased activity in the dorsal medial frontal gyri (BA 6/8) as well as a down-regulation of amygdala activity (Ochsner et al., 2002; Ochsner and Gross, 2005).

In contrast to one of our hypotheses, our results revealed amygdala deactivations for beginners relative to experienced meditators during the processing of emotional stimuli in the Mindful condition. This finding suggests that beginners may have actually taken a more active strategy to down-regulate emotion during the Mindful condition. Indeed, they may have been unable to refrain from automatic judgment of the stimuli and subsequently needed to apply mindfulness after an initial appraisal. This would have turned mindfulness into reappraisal, and may have been reflected in the increased activity in higher-order prefrontal brain areas during the Mindful condition.

Interestingly, the mindfulness-induced amygdala deactivation observed in beginners was not specific to negative pictures, but was also observed during positive emotional processing. In line with this finding, a number of brain imaging studies having found activation of the amygdala in healthy individuals in response to aversive stimuli (see, for instance, Ochsner et al., 2002, 2004), but also to positive emotional stimuli (Beauregard et al., 2001; Sergerie et al., 2008), indicating that this cerebral structure may not be specific to the detection of aversive information but to generally relevant motivational information. Moreover, our findings indicate that during the processing of positive emotional stimuli, mindfulness induced deactivation in brain areas involved in addiction, awareness of interoceptive states of urges and pleasure, and reactivity to positive stimuli (amygdala, hippocampus, insula, basal ganglia; Craig, 2004; Everitt et al., 1999; Haruno et al., 2004; Naqvi and Bechara, 2010; Phillips and Ledoux, 1992; Prado-Alcala and Wise, 1984). The fact that these mindfulness-induced deactivations were observed in beginners, but not in experienced meditators, further supports the notion that beginners were actively down-regulating their emotional responses.

Moreover, the fact that mindfulness did not influence amygdala responses for experienced meditators during emotional processing also supports the interpretation that participants with extensive mindfulness training adopted an accepting perspective towards their emotional responses and allowed themselves to fully experience them. Thus, while experienced meditators may have had some degree of amygdala reactivity to emotional stimuli in the mindful state, the MPFC (BA 10) deactivations during the Mindful condition indicates that mindfulness altered what happened after their initial response (cognitive elaboration, judgments, etc.), which led to decreased subjective emotional intensity. From this perspective, mindfulness may constitute an emotion regulatory strategy that targets cognitive processes related to the acceptance, interpretation and evaluation of emotional responses rather than voluntary emotional inhibition or modulation. This interpretation is highly compatible with the notions taught in the practice of mindfulness, such as acknowledging thoughts and feelings and observing them as temporarily passing events of the mind (Wallace and Shapiro, 2006). As a result, emotions dissipate more rapidly and cognitive resources can become available to the next event arising in the present moment.

Finally, across all conditions and emotional categories, beginners exhibited increased activity in the PCC (BA 29/31), left OFC (BA 47), hippocampus, thalamus, as well as the left fusiform and lingual gyri (BA 17/18/37). Given the involvement of the OFC (BA 47) in linguistic semantic processes (Dapretto and Bookheimer, 1999), the hippocampus in emotional context conditioning (Phillips and Ledoux, 1992), and the PCC in emotional processing as well as emotional memory retrieval (Kober et al., 2008; Maddock et al., 2003), beginners may have had a general tendency to attribute semantic meaning to the pictorial stimuli, which may have triggered enhanced emotional memory retrieval throughout the viewing of the pictures relative to experienced meditators. This would be consistent with the fact that mindfulness is associated with present-moment awareness, without referring to past experiences (Bishop, 2004), and may indicate general neural functional changes associated with extensive mindfulness practice. In addition, increased activity in visual areas in the ventral processing stream have also been reported in a number of studies examining emotional processing using visual stimuli (Kober et al., 2008; Gross, 2007), which may indicate general enhanced attention, saliency, and visual processing to environmental stimuli for beginners compared with experienced meditators. Future studies directly examining the relationship between meditation and visual processing are needed to validate this hypothesis.

\section{Limitations and future directions}

Some limitations of this study have to be acknowledged. First, there may have been differences between the two groups of participants other than mindfulness training (e.g., lifestyle, temperamental predispositions). However, our main objective was to study the effect of long-term mindfulness meditation experience, and studying the effects of more than $1000 \mathrm{~h}$ of mindfulness practice would have been unrealistic or very challenging using a longitudinal design. Nonetheless, future longitudinal studies in larger samples examining the relationship between mindfulness, brain function and emotional processing should be conducted in order to corroborate the findings from this study. Second, due to technical constraints, we were unable to acquire other measures of emotional reactivity, such as galvanic skin response. Ideally, future studies of the impact of mindfulness on emotional processes should include such objective measures in order to understand the influence of mindfulness on physiological responses to emotional stimuli.

\section{Conclusions}

These results indicate that extensive mindfulness practice promotes emotional stability and modulates the activity in a number of 
brain regions implicated in the neural responses to emotional stimuli. Our results also demonstrate that for individuals with long-term practice of meditation, mindfulness induces a pattern of brain activity associated with acceptance of emotional stimuli and reduced interference from internal states, possibly reflecting enhanced present-moment awareness. Thus, as opposed to beginners who exhibited mindfulness-induced patterns of brain activity related to the voluntary regulation of emotion, the findings of this study indicate that a 'lack of control' over emotional states, i.e. acceptance, is associated with the emotional stability achieved by the long-term practice of mindfulness meditation. The findings obtained in this study contribute to the understanding of the relationship between mindfulness meditation, brain function, and emotional processing, and have implications for clinical psychological disorders related to affect and anxiety.

\section{Acknowledgments}

This work was supported by a grant from National Sciences and Engineering Research Council of Canada (NSERC) to M.B. We thank the staff of the Unite de Neuroimagerie Fonctionnelle (UNF) of the Centre de recherche de l'Institut Universitaire de Gériatrie de Montréal (CRIUGM) for their technical assistance.

\section{References}

Amaral, D.G., Price, J.L., Pitkanen, A., Carmichael, S.T., 1992. Anatomical Organization of the Primate Amygdaloid Complex. Wiley-Liss, New York, NY, USA

Arch, J.J., Craske, M.G., 2006. Mechanisms of mindfulness: emotion regulation following a focused breathing induction. Behav. Res. Ther. 44 (12), 1849-1858.

Baer, R.A., 2003. Mindfulness training as a clinical intervention: a conceptual and empirical review. Clin. Psychol. Sci. Pract. 10 (2), 125-143.

Bærentsen, K.B., Stødkilde-Jørgensen, H., Sommerlund, B., Hartmann, T., DamsgaardMadsen, J., Fosnæs, M., Green, A.C., 2010. An investigation of brain processes supporting meditation. Cogn. Process. 11, 57-84.

Beauregard, M., Lévesque, J., Bourgoin, P., 2001. Neural correlates of conscious selfregulation of emotion. J. Neurosci. 21, 1-6.

Bishop, R.S., 2004. Mindfulness: a proposed operational definition. Clin. Psychol. Sci. Pract. 11 (3), 230-241.

Bondolfi, G., Jermann, F., der Linden, M.V., Gex-Fabry, M., Bizzini, L., Rouget, B.W., MyersArrazola, L., Gonzalez, C., Segal, Z., Aubry, J.M., Bertschy, G., 2010. Depression relapse prophylaxis with Mindfulness-Based Cognitive Therapy: replication and extension in the Swiss health care system. J. Affect. Disord. 122 (3), 224-231.

Brefczynski-Lewis, J.A., Lutz, A., Schaefer, H.S., Levinson, D.B., Davidson, R.J., 2007. Neural correlates of attentional expertise in long-term meditation practitioners. Proc. Natl. Acad. Sci. U. S. A. 104 (27), 11483-11488.

Broderick, P.C., 2005. Mindfulness and coping with dysphoric mood: contrasts with rumination and distraction. Cogn. Ther. Res. 29 (5), 501-510.

Buckner, R.L., Andrews-Hanna, J.R., Schacter, D.L., 2008. The brain's default network: anatomy, function, and relevance to disease. Ann. N. Y. Acad. Sci. 1124, 1-38.

Cohen, J., 1992. A power primer. Psychol. Bull. 112 (1), 155-159.

Craig, A.D., 2004. Human feelings: why are some more aware than others? Trends Cogn. Sci. 8 (6), 239-241.

Creswell, J.D., Way, B.M., Eisenberger, N.I., Lieberman, M.D., 2007. Neural correlates of dispositional mindfulness during affect labeling. Psychosom. Med. 69 (6), 560-565.

Critchley, H.D., Wiens, S., Rotshtein, P., Ohman, A., Dolan, R.J., 2004. Neural systems supporting interoceptive awareness. Nat. Neurosci. 7 (2), 189-195.

Dapretto, M., Bookheimer, S.Y., 1999. Form and content: dissociating syntax and semantics in sentence comprehension. Neuron 24 (2), 427-432.

Davidson, R.J., 1998. Anterior electrophysiological asymmetries, emotion, and depression: conceptual and methodological conundrums. Psychophysiology 35 (5), 607-614.

Everitt, B.J., Parkinson, J.A., Olmstead, M.C., Arroyo, M., Robledo, P., Robbins, T.W., 1999 Associative processes in addiction and reward. The role of amygdala-ventral striata subsystems. Ann. N. Y. Acad. Sci. 877, 412-438.

Farb, N.A., Segal, Z.V., Mayberg, H., Bean, J., McKeon, D., Fatima, Z., Anderson, A.K., 2007. Attending to the present: mindfulness meditation reveals distinct neural modes of self-reference. Soc. Cogn. Affect. Neurosci. 2 (4), 313-322.

Farb, N.A., Anderson, A.K., Mayberg, H., Bean, J., McKeon, D., Segal, Z.V., 2010. Minding one's emotions: mindfulness training alters the neural expression of sadness. Emotion 10 (1), 25-33.

Friston, K.J., Holmes, A., Poline, J.B., Price, C.J., Frith, C.D., 1996. Detecting activations in PET and fMRI: levels of inference and power. Neuroimage 4 (3 Pt 1), 223-235.

Goldin, P.R., Gross, J.J., 2010. Effects of mindfulness-based stress reduction (MBSR) on emotion regulation in social anxiety disorder. Emotion 10 (1), 83-91.

Grant, J.A., Courtemanche, J., Rainville, P., 2011. A non-elaborative mental stance and decoupling of executive and pain-related cortices predicts low pain sensitivity in Zen meditators. Pain 152 (1), 150-156.
Gross, J.J., 2007. Handbook of Emotion Regulation. The Guilford Press, New York, NY, USA.

Haruno, M., Kuroda, T., Doya, K., Toyama, K., Kimura, M., Samejima, K., Imamizu, H., Kawato, M., 2004. A neural correlate of reward-based behavioral learning in caudate nucleus: a functional magnetic resonance imaging study of a stochastic decision task. J. Neurosci. 24 (7), 1660-1665.

Hills, P., Argyle, M., 2001. Emotional stability as a major dimension of happiness. Pers. Individ. Diff. 31 (8), 1357-1364.

Kabat-Zinn, J., 1994. Wherever You Go There You Are. Hyperion Books, New York, NY, USA.

Kabat-Zinn, J., Massion, A.O., Kristeller, J., Peterson, L.G., Fletcher, K.E., Pbert, L., Lenderking, W.R., Santorelli, S.F., 1992. Effectiveness of a meditation-based stress reduction program in the treatment of anxiety disorders. Am. J. Psychiatry 149 (7), 936-943.

Kim, Y.W., Lee, S.H., Choi, T.K., Suh, S.Y., Kim, B., Kim, C.M., Cho, S.J., Kim, M.J., Yook, K., Ryu, M., Song, S.K., Yook, K.H., 2009. Effectiveness of mindfulness-based cognitive therapy as an adjuvant to pharmacotherapy in patients with panic disorder or generalized anxiety disorder. Depress. Anxiety 26 (7), 601-606.

Kober, H., Barrett, L.F., Joseph, J., Bliss-Moreau, E., Lindquist, K., Wager, T.D., 2008. Functional grouping and cortical-subcortical interactions in emotion: a metaanalysis of neuroimaging studies. Neuroimage 42 (2), 998-1031.

Kross, E., Davidson, M., Weber, J., Ochsner, K., 2009. Coping with emotions past: the neural bases of regulating affect associated with negative autobiographical memories. Biol. Psychiatry 65 (5), 361-366.

Lang, P.J., Bradley, M.M., Cuthbert, B.N., 2005. International Affective Picture System (IAPS): Instruction Manual and Affective Ratings, Technical Report A-6. The Center for Research in Psychophysiology, University of Florida.

Levesque, J., Eugene, F., Joanette, Y., Paquette, V., Mensour, B., Beaudoin, G., Leroux, J.M., Bourgouin, P., Beauregard, M., 2003. Neural circuitry underlying voluntary suppression of sadness. Biol. Psychiatry 53 (6), 502-510.

Lutz, A., Brefczynski-Lewis, J., Johnstone, T., Davidson, R.J., 2008. Regulation of the neural circuitry of emotion by compassion meditation: effects of meditative expertise. PLoS One 3 (3), e1897 1-10.

Maddock, R.J., Garrett, A.S., Buonocore, M.H., 2003. Posterior cingulate cortex activation by emotional words: fMRI evidence from a valence decision task. Hum. Brain Mapp. 18 (1), 30-41.

Mennin, D.S., Heimberg, R.G., Turk, C.L., Fresco, D.M., 2005. Preliminary evidence for an emotion dysregulation model of generalized anxiety disorder. Behav. Res. Ther. 43 (10), 1281-1310

Naqvi, N.H., Bechara, A., 2010. The insula and drug addiction: an interoceptive view of pleasure, urges, and decision-making. Brain Struct. Funct. 214 (5-6), 435-450.

Nataraja, S., 2008. The Blissful Brain Neuroscience and Proof of the Power of Meditation. Octopus Publishing Group Ltd., New York, NY, USA.

Newberg, A., Alavi, A., Baime, M., Pourdehnad, M., Santanna, J., d'Aquili, E., 2001. The measurement of regional cerebral blood flow during the complex cognitive task of meditation: a preliminary SPECT study. Psychiatry Res. 106 (2), 113-122.

Norris, C.J., Larsen, J.T., Cacioppo, J.T., 2007. Neuroticism is associated with larger and more prolonged electrodermal responses to emotionally evocative pictures. Psychophysiology 44 (5), 823-826.

Ochsner, K.N., Gross, J.J., 2005. The cognitive control of emotion. Trends Cogn. Sci. 9 (5), 242-249.

Ochsner, K.N., Bunge, S.A., Gross, J.J., Gabrieli, J.D., 2002. Rethinking feelings: an FMRI study of the cognitive regulation of emotion. J. Cogn. Neurosci. 14 (8), 1215-1229.

Ochsner, K.N., Ray, R.D., Cooper, J.C., Robertson, E.R., Chopra, S., Gabrieli, J.D., Gross, J.J., 2004. For better or for worse: neural systems supporting the cognitive down- and up-regulation of negative emotion. Neuroimage 23 (2), 483-499.

Phan, K.L., Wager, T., Taylor, S.F., Liberzon, I., 2002. Functional neuroanatomy of emotion: a meta-analysis of emotion activation studies in PET and fMRI. Neuroimage 16 (2), 331-348.

Phillips, R.G., LeDoux, J.E., 1992. Differential contribution of amygdala and hippocampus to cued and contextual fear conditioning. Behav. Neurosci. 106 (2), 274-285.

Prado-Alcala, R., Wise, R.A., 1984. Brain stimulation reward and dopamine terminal fields. I. Caudate-putamen, nucleus accumbens and amygdala. Brain Res. 297 (2), 265-273.

Ramel, W., Goldin, P.R., Carmona, P.E., McQuaid, J.R., 2004. The effects of mindfulness meditation on cognitive processes and affect in patients with past depression. Cogn. Ther. Res. 28 (4), 433-455.

Ricard, M., 2008. L'art de la Méditation. Nil éditions, Paris, France.

Sergerie, K., Chochol, C., Armony, J.L., 2008. The role of the amygdala in emotional processing: a quantitative meta-analysis of functional neuroimaging studies. Neurosci. Biobehav. Rev. 32 (4), 811-830.

Sheline, Y.I., Barch, D.M., Price, J.L., Rundle, M.M., Vaishnavi, S.N., Snyder, A.Z., Mintun, M.A., Wang, S., Coalson, R.S., Raichle, M.E., 2009. The default mode network and self-referential processes in depression. Proc. Natl. Acad. Sci. U. S. A. 106 (6), 1942-1947.

Talairach, J., Tournoux, P., 1988. Stereotaxic localization of central gray nuclei. Neurochirurgia (Stuttg) 1 (1), 88-93.

Teasdale, J.D., Segal, Z.V., Williams, J.M., Ridgeway, V.A., Soulsby, J.M., Lau, M.A., 2000. Prevention of relapse/recurrence in major depression by mindfulness-based cognitive therapy. J. Consult. Clin. Psychol. 68 (4), 615-623.

Thich Nhat Hanh, 1994. Le Miracle de la Pleine Conscience. L'espace bleu, France.

Wallace, B.A., Shapiro, S.L., 2006. Mental balance and well-being: building bridges between Buddhism and Western psychology. Am. Psychol. 61 (7), 690-701.

Zeidan, F., Johnson, S.K., Gordon, N.S., Goolkasian, P., 2010. Effects of brief and sham mindfulness meditation on mood and cardiovascular variables. J. Altern. Complement Med. 16 (8), 867-873. 